STUDI

FRANCESI

\section{Studi Francesi}

Rivista quadrimestrale fondata da Franco Simone

180 (LX | III) | 2016

Varia

\title{
Textes au corps. Promenades et musardises sur les terres de Marie Madeleine Fontaine, éds. Didier Kahn, Elsa Kammerer, Anne-Hélène Klinger-Dollé, Marine Molins et Anne-Pascale Pouey-Mounou
}

\section{Maurizio Busca}

\section{(2) OpenEdition}

\section{Journals}

Edizione digitale

URL: http://journals.openedition.org/studifrancesi/5276

DOI: 10.4000/studifrancesi.5276

ISSN: 2421-5856

Editore

Rosenberg \& Sellier

Edizione cartacea

Data di pubblicazione: 1 dicembre 2016

Paginazione: 513

ISSN: 0039-2944

\section{Notizia bibliografica digitale}

Maurizio Busca, "Textes au corps. Promenades et musardises sur les terres de Marie Madeleine Fontaine, éds. Didier Kahn, Elsa Kammerer, Anne-Hélène Klinger-Dollé, Marine Molins et Anne-Pascale PoueyMounou », Studi Francesi [Online], 180 (LX | III) | 2016, online dal 01 janvier 2017, consultato il 18 septembre 2020. URL : http://journals.openedition.org/studifrancesi/5276 ; DOI : https://doi.org/ 10.4000/studifrancesi.5276

Questo documento è stato generato automaticamente il 18 settembre 2020.

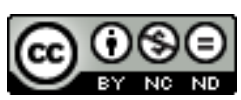

Studi Francesi è distribuita con Licenza Creative Commons Attribuzione - Non commerciale - Non opere derivate 4.0 Internazionale. 
Textes au corps. Promenades et musardises sur les terres de Marie Madeleine Fontaine, éds. Didier Kahn, Elsa Kammerer, Anne-Hélène Klinger-Dollé, Marine Molins et Anne-Pascale Pouey-Mounou

\author{
Maurizio Busca
}

\title{
NOTIZIA
}

Textes au corps. Promenades et musardises sur les terres de Marie Madeleine Fontaine, études réunies par Didier KAHN, Elsa KAMMERER, Anne-Hélène KLINGER-DOLLÉ, Marine MOLINS et Anne-Pascale PouEY-MOUNOU, Genève, Droz, 2015, «Travaux d'Humanisme et Renaissance» DL, 526 pp.

Il volume, pubblicato in onore della grande specialista del Rinascimento francese Marie Madeleine Fontaine, riunisce ventotto contributi concernenti tematiche cui la studiosa ha consacrato larga parte delle sue ricerche. In particolare, oltre ai suoi lavori fondamentali sulla poesia del Cinquecento, le riflessioni suscitate in occasione di due importanti convegni da lei organizzati nel 1987 e nel 2003 (Le Corps à la Renaissance e Rire à la Renaissance) trovano una evidente continuità nella presente raccolta. All'interno di questa, si definisce un percorso che, muovendo dall'osservazione del corpo umano, delle sue funzioni, delle sue parti anatomiche e delle loro proprietà (cap. I), ne esplora la dinamicità: il cap. II è infatti dedicato alle rappresentazioni del corpo in movimento e dell'esercizio fisico, sia esso addestramento militare o allenamento sportivo. I due capitoli successivi guardano invece ai viaggi (promenades, esplorazioni di 
terre note e ignote) e agli animali che popolano le pagine dei trattati di storia naturale e delle opere narrative. Negli ultimi tre capitoli l'asse di interesse si orienta su questioni concernenti la riscoperta (materiale o metaforica) di testi creduti persi o trascurati dalla critica; sui legami fra poesia e musica e fra giochi testuali e giochi musicali; infine, sui rapporti (anche di carattere ludico) fra poeti antichi studiati o tradotti da autori del Cinquecento, e fra questi ultimi e i loro lettori ottocenteschi. Nell'impossibilità di fornire in questa sede un resoconto adeguato del contenuto dei numerosi articoli raccolti in questa ricchissima miscellanea, se ne fornirà unicamente l'indice.

2 Chapitre premier («Des parties du corps, des humeurs et des sens»): Richard COOPER, «Ad formam nasi cognoscitur ad te levavi» ("Gargantua", chap. XL). Rabelais à la Foire des Nez, pp.15-31; Elsa KAMMERER, Toute de miel. La Gargamelle de Johann Fischart (1575-1590), pp. 33-49; Danielle JACQUART, Médecine ou/et alchimie: l'étrange régime d'Angelo de Aquila (1415), pp. 51-66; Max ENGAMMARE, «Avoir les oreilles dressées à la Parole de Dieu». L'ouïe chez Jean Calvin, pp. 67-79; Anne-Hélène KLINGER-DOLLÉ, Le corps du sage et la notion d'art (ars) dans la philosophie de Charles de Bovelles (1479-1567), pp. 81-96; Sylvie DESWARTE-ROSA, L'Adam mélancolique de Francisco de Holanda, pp.97-128; Anne-Pascale POUEY-MOUNOU, Brocards, pastiches et mélanges: bas morceaux choisis des "Epithetes" de La Porte, pp. 129-145; Chapitre II («Des exercices physiques, de la guerre et des jeux»): Jean-Louis FouRnEL, Le corps du soldat chez Machiavel, pp. 149-162; François CORNILLIAT, Le corps intermittent: Louis de La Trémoille entrevu par Jean Bouchet, pp.163-172; Guy BоnномmE, La géométrie des chasses contre l'arithmétique des points dans le "Trattato del giuoco della palla d'Antonio Scaino" (1555), pp.173-183; Ginette VAGENHEIM, La représentation dissidente du corps à l'époque de la Contre-Réforme: un dessin inédit de saint Jérôme par Pirro Ligorio (1512-1583), pp. 185-198; Chapitre III («Des promenades, voyages et terres pérégrines»): Jean CÉARD, De Budé à Aneau: lectures de l'“Utopie" de Thomas More, pp. 201-213; Nada GRUJIC', À la recherche de l'Arcadie ragusaine, pp. 215-225; Rosanna GORRIS CAMOS, "Je và et vien par volontaire fuite»: La Savoye en bleu, en vert, en noir, pp. 227-252; Frank LESTRINGANT, Faut-il expier la Renaissance? L'ouverture anthropologique du XVI siècle, pp. 253-270; Hope GLIDDEN, La Renaissance de Gustave Flaubert, pp. 271-287; Chapitre IV («Des bêtes volatiles, marines et fabuleuses»): Michel JOURDE, Alectryomancie dans la basse-cour: une page du "Discours oeconomique" de Prudent Le Choyselat (1569), pp. 291-304; Stephen BAMFORTH, Monstre, mer et merveille à la Renaissance, pp. 305-326; Didier KAHN, Les lectures alchimiques du roman de "Perceforest" et de la «beste glatissante», pp. 327-343; Chapitre V («De la redécouverte des livres»): Catherine MAGNIEN-SIMONIN, Le dernier voyage de Jeanne d'Albret en 1572 (Jean de La Gessée, "Recueil des Letres missives, Discours et Harangues familiaires", Paris, Jean de Lastre, 1579), pp. 347-363; Jean BALSAMO, Le frontispice des "Essais" (1588): Montaigne et ses décors, pp. 365-379; Chapitre VI («Chansons et facéties»): Henri VANHULST, Comment, d'après une chanson anonyme de 1529, confondre une femme adultère, pp. 383-393; Marie-Alexis CoLIN, Ung hu, deux hu, troix hurons de villaige: jeux de corps, jeux de mots et jeux musicaux dans une chanson de Benedictus Appenzeller (1544), pp.395-409; Annie COEURDEVEY, Janequin vs Fresneau: double attribution, double version du «joly jeu», pp. 411-423; Frank DoBBINS, Notes sur Rémy Belleau et ses musiciens, pp. 425-462; Chapitre VII («Poètes et poésies»), François RIGolot, Nomen, numen: jeux d'enfants dans quelques épitaphes joyeuses de Marot, pp. 465-471; Marine MoLINS, La traduction à bras-le-corps: fragments de l'“Enéide" par Du Bellay, pp. 473-486; Sylvie THOREL, Baudelaire «chétif», pp. 487-499. 